SACHBERICHT

\section{Leistungen bilanzieren, Erfolge belegen}

\author{
Horst Bossong
}

Sachberichte gelten bei vielen sozialen Organisationen als ungeliebte Pflichtübung. Was dabei oft übersehen wird, ist die Tatsache, dass der Sachbericht als solide Leistungsbilanz fungieren kann, die als publizitätswirksame Darstellung die Kompetenz des Unternehmens dokumentiert. Sachberichte stellen zudem das Fundament für eine sachgerechte politische Steuerung und Planung sozialer Infrastruktur dar.

Im Grunde genommen ist es eine pure Selbstverständlichkeit: Wer mit öffentlichen Mitteln wirtschaftet, hat Rechenschaft abzulegen, und zwar so, dass in überzeugender Weise deutlich wird, ob und wie die eingesetzten Mittel zweckmäßig, wirtschaftlich und wirksam verwandt wurden (Piduch 2005).

Dennoch gelten das Erstellen und die Prüfung von Sachberichten vielfach bis heute für alle Beteiligten als eine ungeliebte Pflichtübung, der man sich, wenn es nur irgendwie ginge, gerne entzöge. Doch sowohl die Bundeshaushaltsverordnung als auch sämtliche Landeshaushaltsordnungen schreiben die Vorlage und Prüfung von Sachberichten zwingend vor (vgl. für den Bund: VV Nr.5.1 zu $\S 44 \mathrm{BHO}$, ANBest-I). Die Frage ist also, was sollen und können Sachberichte leisten und wie sollten sie ausgestaltet sein.

Nach hergebrachter Auffassung besteht die Funktion der Sachberichte vorrangig in der Legitimation der zweckmäßigen und wirtschaftlichen Mittelverwendung gegenüber dem öffentlichen Finanzier. Dies implizierte in der Vergangenheit und zu einem
Gutteil noch heute eine überwiegend defensiv-rechtfertigende Vorgehensweise: Was Not tut, weil es verwaltungsseitig verlangt wird, wird berichtet, wo immer es zur Sicherung weiterer Zuschüsse erforderlich und zweckdienlich erscheint, werden Ergebnisse und statistische Daten argumentativ ins rechte Licht gerückt und nicht selten beschönigend dargestellt. Darüber hinaus gehende Ansprüche werden üblicherweise mit dem Sachbericht nicht verbunden. (Der Autor hat in SOZIALwirtschaft 6/2006 am Beispiel eines Jahresberichts einer Drogenhilfeeinrichtung gezeigt, wie Leistungsberichte nicht aussehen sollten. - Anmerkung der Redaktion)

Was mithin oft völlig übersehen oder ignoriert wird, ist die Tatsache, dass der Sachbericht als eine solide Leistungsbilanz fungieren soll, mittels derer eine fundierte Qualitätsbewertung und Erfolgskontrolle der geleisteten Arbeit möglich werden soll (Krämer/Schmidt 2005: A II und E I). Erst und nur damit bildet er das Fundament für eine sachgerechte politische Steuerung und Planung sozialer Infrastruktur.

Allerdings begegnen dementsprechend die Sachberichte und die ihnen zugrunde liegende Dokumentationen hohen Anforderungen, speziell die Qualitätsbewertung sozialarbeiterischer Aktivitäten - sie ist immerhin die Voraussetzung für jede Ziel führende Steuerung - gilt dabei gemeinhin als äußerst schwierig (etwa: Bauer 2001; Olk u. a. 2004; Rose 2004; Schaarschuh 2003).

Widerstände hiergegen haben eine entsprechend lange Tradition. Dies gilt vor allem mit Blick auf Verfahren der quantitativen Erfolgsmessung, die vielfach als verwaltungsseitig oktroy-

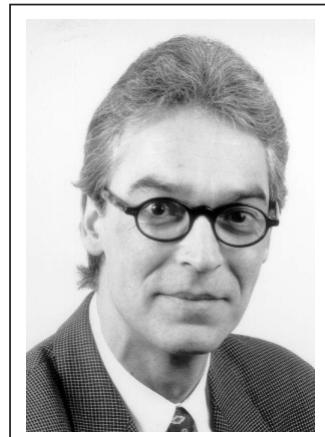

Prof. Dr. Horst Bossong (56) lehrt an der Universität DuisburgEssen Verwaltungs-

wissenschaften mit dem Schwerpunkt Sozialverwaltung, Drogen- und Drogenhilfepolitik. Von 1989 bis 1999 war der studierte Sozialarbeiter und Sozialwissenschaftler Drogenbeauftragter des Senats der Freien und Hansestadt Hamburg. E-Mail horst.bossong@uni-essen.de

iert und rein »betriebswirtschaftlichen Konzepten « gehorchend kritisiert werden (Merchel 2000; Peters 2004). Dennoch: Qualitätsbewertung und im Gefolge eine rationale Planung anhand von Dokumentationen ist durchaus möglich, jedenfalls dann, wenn die Dokumentation bestimmten Minimalstandards, wie sie vor allem in den Wirtschaftswissenschaften propagiert werden, entspricht und wenn sie durch - ebenfalls von Ökonomen postulierten Kundenbefragungen und andere qualitative Erhebungen ergänzt wird (Bruhn 2004; Ebel 2003).

\section{Systematisierungen}

Die Probleme, mit denen man es bei einer durch Sachberichte ermöglichten Leistungsbewertung und Leistungsbilanzierung zu tun hat, beginnen häufig damit, dass entscheidende Bezugspunkte unklar sind.

Wer soziale Dienstleistungen bilanzieren will, muss notwendig zunächst für jede im Einzelnen zu erbringende oder erbrachte Leistung die konzeptionell damit jeweils verfolgten Ziele kennen und benennen, und er muss wissen und angeben, mit welchen Mitteln die jeweiligen Ziele und Teilziele in welchen Zeitfristen und mit welchen längerfristig zu erwartenden Effekten erreicht werden sol- 


\section{Wie Sachberichte aussehen sollen}

Die qualifizierte Struktur eines Sachberichts kann auf vier Pfeilern ruhen:

1. Benennung der im Zuwendungsbescheid beschriebenen Förderzwecke

1.1. Beschreibung der übergeordneten strategischen (Langfrist-) Ziele

1.2. Benennung der Rahmenbedingungen (z. B. gesetzliche Grundlagen)

2. Dokumentation der realisierten Aufgabenschwerpunkte, der erbrachten Leistungen und der erzielten Ergebnisse (unterteilt in einzelne Leistungsbereiche)

2.1. Leistungsbereich 1:

2.1.1. Benennung der planmäßig vorgesehenen Leistungen nach Prioritätssetzung einschließlich der jeweiligen operativen Zieldefinition; Personalausstattung sowie Angaben zur Qualifikation, gegebenenfalls räumlich-sächliche Ausstattung.

Motto: Was sollte mit welchen Ressourcen wie gemacht werden?

2.1.2. Statistische Leistungsdokumentation: Abgleich der Soll-Ist-Kennziffern anhand statistischer Daten mit Rekurs auf die jeweiligen Ziele und Teilziele (gegebenenfalls im mehrjährigen Zeitverlauf); soweit leistungsbereichsbezogen er- folgt: Dokumentation durchgeführter Kundenund Mitarbeiterbefragungen.

Motto: Was wurde tatsächlich mit welchen Ergebnissen wie gemacht?

2.1.3. Ergebnisbewertung: Benennung der erfolgreichen sowie der im Hinblick auf die Zweckerfüllung ganz oder teilweise abgeschlossenen Leistungssegmente und Aufgabenbereiche; Analyse des Grades der erfolgreichen Zielerreichung.

Motto: Welche Ziele oder Teilziele sind mit welchem Resultat (gegebenenfalls wie nachhaltig) erreicht worden?

2.1.4. Benennung wichtiger Schwachstellen und Defizitfelder; Analyse und Diskussion der möglichen Gründe und Ursachen; Beschreibung der für notwendig erachteten Zielrevision und erforderlichen Umstrukturierungen.

Motto: Was haben wir warum falsch gemacht und wie wollen wir es besser machen?

2.2. Leistungsbereich 2: (entsprechend 2.1.1. bis 2.1.4.)

3. Bereichsübergreifende Leistungen (z. B. Kooperationen und Vernetzungsbemühungen mit komplementären Institutionen; übergreifende Maßnahmen zur Qualitätssicherung)

4. Zusammenfassung der wichtigsten Ergebnisse und der geplanten Vorhaben für den kommenden Berichtszeitraum

Horst Bossong len. Kennt oder benennt er weder die genauen Ziele und die zum Berichtszeitpunkt erreichte Zielverwirklichung noch den vorher geplanten und dann tatsächlich erfolgten Mitteleinsatz, dann wird eine seriöse Bilanzierung letztlich unbefriedigend bleiben oder gar komplett scheitern (Schmalen 2002: 710 ff.).

Vorausgesetzt ist damit eine entsprechend gut fundierte und stets auf dem neuesten Stand gehaltene Konzeption der zu beurteilenden Einrichtung, die die verschiedenen Ziele und Teilziele sowie die für die Zielerreichung nötigen (Einzel-) Leistungen und den dementsprechenden Mitteleinsatz zu benennen hätte. Eine Vorbedingung, die in vielen sozialen Einrichtungen selbst heute noch alles andere als selbstverständlich zu sein scheint. Vor allem die im sozialarbeiterischen Sektor vielfach übliche Praxis, Projekte über viele Jahre nahezu unverändert zu fördern, also weitgehend auf eine regelmäßige Überplanung zu verzichten, begünstigt den Verzicht auf konzeptionelle Anpas- sungen und Restrukturierungen, die Fördermittel fließen auf Basis des in der Regel einmalig vor Projektbeginn vorgelegten Konzepts meist ohne prozessbegleitendes behördliches Fachcontrolling - eine Praxis, die mit den haushaltsrechtlichen Vorschriften freilich nur schwer in Einklang zu bringen (Krämer/Schmidt 2005: D VI; Nr.3) und jedenfalls allemal mit den Ansprüchen an modernes Qualitätsmanagement gänzlich unvereinbar ist (Bruhn 2004).

Hilfreich für eine qualitativ anspruchsvolle Leistungsbilanzierung sind überdies eine sachbezogene Systematisierung sowie eine Priorisierung der verschiedenen Einzelleistungen, die in einer Einrichtung erbracht werden. Konzeptionell verdeutlicht und im Sachbericht dokumentiert werden muss also, welche der verschiedenen, oft zahlreichen (Einzel-) Leistungen inhaltlich und systematisch zusammengehören (also etwa: Beratungs- und Betreuungsleistungen, Service- und Versorgungsleistungen usw.). Parallel dazu sollte erkenn- bar sein, welche Leistungen oder Leistungspakete verbunden mit welchen Zielsetzungen im Vordergrund der Einrichtung stehen und Priorität besitzen. Geht es also beispielsweise in einer Einrichtung eher um pädagogische Betreuungs- oder um soziale Versorgungsleistungen, eher um geschützte Aufenthalts- oder um gezielte Förderangebote, und (wie) lassen sich hier sodann jeweils geeignete Zielhierarchien, die sukzessive erreicht werden sollen, definieren?

Von den primären Leistungen abzugrenzen wären die komplementären oder Sekundärleistungen und Sekundärziele, die der primären Zielsetzung Nachdruck verleihen und ihr in besonders geeigneter Weise zum Durchbruch verhelfen (sollen). Als conditio sine qua non unterstellt wird dem gemäß, dass jede Leistung einem konkret beschreibbaren Ziel zuzuordnen ist, es mit anderen Worten keine Leistung ohne Ziel geben darf. Im Auswertungsprozess selbst muss schließlich, das liegt auf der Hand, unterschieden werden zwi- 
schen harten und weichen Indikatoren. Zu den harten Indikatoren, die meistens leicht mess- und berechenbar sind, gehören beispielsweise die räumlich-sächliche sowie die personelle Ausstattung, die Öffnungszeiten, Erreichbarkeit und Frequentierung, die Auslastung, die Anzahl der pädagogischen Gespräche und sonstigen Interventionen, gegebenenfalls die Abbrecherquote und ähnliches mehr. Speziell hier sind, um zu einer sachgerechten Gesamtbewertung zu kommen, Vergleiche mit ähnlichen Angeboten (also Benchmarking) häufig hilfreich und nötig.

Weiche Indikatoren sind demgegenüber zweifellos schwerer zu benennen, allerdings keineswegs unerfassbar. Hier geht es vielfach um eher subjektive Einschätzungen oder Resultate, die erst nach einer längeren zeitlichen Frist und im Zusammenwirken mit anderen Faktoren wirksam werden. Dies betrifft beispielsweise die sehr komplexen Fragen nach der Zufriedenheit der Nutzer mit den Angeboten, nach dem Lernoder Sozialisierungserfolg, dem praktischen Nutzen für den Alltag des Klienten und schließlich nach dem ökonomischen Erfolg und

Gemeinnutzen - Modelle zur Evaluation komplexerer Wirkungszusammenhänge stehen durchaus zur Verfügung (Arnold/ Pätzhold 2004; Hoettermann 2004), sie müssten nur regelhaft genutzt werden.

\section{Bilanzpolitik und Bilanzanalyse}

Es ist keine Frage: Mit Bilanzen will man glänzen und nicht scheitern. Doch wer seine Schwächen, Fehler und Misserfolge verschleiert, ungünstige Fakten unterdrückt, kurz die Ergebnisse beschönigt und »frisiert «, der, so jedenfalls die Usancen in der Privatwirtschaft, macht sich gegebenenfalls der Bilanzfälschung schuldig. Im öffentlich finanzierten Bereich sieht dies im Grunde nicht anders aus - im Zweifel werden gezahlte Zuwendungen zurückgefordert, das bezuschusste Projekt nicht weiter gefördert und schlimmstenfalls Strafverfahren gegen die Verantwortlichen eingeleitet. Und doch liegt es auf der Hand und ist verständlich, dass mit dem Sachbericht Leistungsstärke, Zuverlässigkeit und Erfolge dokumentiert, also insgesamt die Reputation und Förderungswür- digkeit gesichert und wenn möglich gesteigert werden sollen. Freilich vernebelt diese Absicht zuweilen den Umstand, dass eine lediglich auf positive Selbstdarstellung zielende Berichterstattung letztlich unbrauchbar und somit hochgradig kritikwürdig ist, weil sie weder hilfreich noch realistisch ist.

\section{Resümee}

Sachberichte sollten immer beides sein: die publizitätswirksame Darstellung und die Sachkompetenz dokumentierende, gründliche Analyse des Erreichten.

Ersteres stellt die Bilanzpolitik, letzteres die Bilanzanalyse dar (Schierenbeck 1999: 581 ff.). Sachlogisch fußt dabei die Bilanzpolitik zwingend auf der vorangegangenen Analyse (Schierenbeck, ebd.; Schmalen 2002: 671 ff.). Diese freilich schließt das Identifizieren, Benennen und Bewerten von Fehlentwicklungen, Misserfolgen und Defiziten ein. Im zuwendungsfinanzierten Sozialbereich ist es allein der Fehlinterpretation der für die Sachberichterstattung maßgeblichen Rechtsnorm zu verdanken, dass das Eingestehen von Fehlern und Schwächen gemeinhin - und zwar sowohl auf Seiten der Einrichtungsbetreiber als auch auf Seiten der fördernden Verwaltung - als Malus bewertet wird. Die Chance zur Verbesserung, zur Neujustierung und konstruktiven Steuerung wird so leichthin vertan.

Zielführend wäre es, Sachberichte nach der Gründlichkeit ihres dokumentarischen (insbesondere statistischen) und analytischen Teils zu goutieren und hierbei in besonderer Weise darauf zu achten, ob und inwieweit Schwachstellen offen gelegt und sodann plausible Nachbesserungs- und Veränderungsvorschläge für die kommende Berichtsperiode unterbreitet werden.

\section{Literatur}

Arnold, R./Pätzhold, H. (2004) Qualitätsstandards in der Erwachsenenbildung. In: Peterander/Speck (Hg.) Qualitätsmanagement in sozialen Einrichtungen. 2. Aufl. München 2004, S. 102-113.

Bauer, R. (2001) Personenbezogene Soziale Dienstleistungen. Opladen.

Bruhn, M. (20045) Qualitätsmanagement für Dienstleistungen. Berlin $u$. a.

Bruhn, M. (2000) Kundenerwartungen. Theoretische Grundlagen, Messung und Managementkonzept. In: Zeitschrift für Betriebswirtschaft, 70. Jg., Nr. 9, S. 1031-1054.

Ebel, B. (2003) Qualitätsmanagement bei Dienstleistungen. In: Pepels, W. (Hg.) Betriebswirtschaft der Dienstleistungen. Herne/Berlin, S. 161-189.

Hoettermann, H., u. a. (2004) Praxisentwicklung und mehrstufige Evaluation in realen Veränderungsprozessen. In: Peters, F./Koch, J. (Hg.) Integrierte erzieherische Hilfen. Weinheim und München; S. 129-148.

Hüllinghorst, R. (2005) Versorgung Abhängigkeitskranker in Deutschland. In: Deutsche Hauptstelle für Suchtfragen (Hg.) Jahrbuch Sucht 2006. Geesthacht, S. 154-169.

Krämer, E./Schmidt, J. (2005) Zuwendungsrecht - Zuwendungspraxis. Kommentar. 71. Lfg., Heidelberg.

Merchel, J. (20042) Qualitätsmanagement in der Sozialen Arbeit. Weinheim und München.

Oehme, W. (2003) Beispiel Beratung. In: Pepels, W. (Hg.) Betriebswirtschaft der Dienstleistungen. Herne/Berlin, S. 420-427.

Olk, Th./Otto, H.-U./Backhaus-Maul, H. (2004) Soziale Arbeit als Dienstleistung, Zur analytischen und empirischen Leistungsfähigkeit eines theoretischen Konzepts. In: dies. (Hg.) Soziale Arbeit als Dienstleistung. München, S. IX-LXXXII.

Peters, F. (2004) Qualitätsentwicklung unter den Bedingungen von Markt und Wettbewerb. In: Beckmann, Ch. u. a. (Hg.) Qualität in der Sozialen Arbeit. S. 155-171, Wiesbaden.

Piduch, E. A. (2005) Bundeshaushaltsordnung. Kommentar. 2. Aufl., 9. Lfg., Stuttgart.

Rose, B. (2004) Wer bestimmt die Qualität. Anmerkungen zum QualitätsDiskurs und seinen Wirkungen in der Sozialen Arbeit. In: Beckmann, C. u. a. (Hg.) Qualität in der Sozialen Arbeit. Wiesbaden, S. 211-220.

Schaarschuh, A. Die Privilegierung des Nutzers. Zur theoretischen Begründung sozialer Dienstleistung. In: Olk, Th./Otto, H.-U. (Hg.) Soziale Arbeit als Dienstleistung. München, S. 150-169.

Schierenbeck, H. (1999) 14. Auflage. Grundzüge der Betriebswirtschaft. München und Wien.

Schmalen, H. (2002) Grundlagen und Probleme der Betriebswirtschaft. Stuttgart. 